\title{
Theta-Frequency Synaptic Potentiation in CA1 In Vitro Distinguishes Cognitively Impaired from Unimpaired Aged Fischer 344 Rats
}

\author{
Geoffrey C. Tombaugh, Wayne B. Rowe, Ana R. Chow, Timothy H. Michael, and Gregory M. Rose \\ Memory Pharmaceuticals Corporation, Montvale, New Jersey 07645
}

Hippocampal-dependent learning and memory deficits have been well documented in aging rodents. The results of several recent studies have suggested that these deficits arise from weakened synaptic plasticity within the hippocampus. In the present study, we examined the relationship between hippocampal long-term potentiation (LTP) in vitro and spatial learning in aged (24-26 months) Fischer 344 rats. We found that LTP induced in the CA1 region using theta-frequency stimulation (5 $\mathrm{Hz}$ ) is selectively impaired in slices from a subpopulation of aged rats that had shown poor spatial learning in the Morris water maze. LTP at $5 \mathrm{~Hz}$ in aged rats that did not show learning deficits was similar to that seen in young (4-6 months) controls. We also found that $5 \mathrm{~Hz}$ LTP amplitude strongly correlated with individual learning performance among aged rats. The difference in $5 \mathrm{~Hz}$ LTP mag-

Learning and memory impairments are among the most commonly recognized psychological features of human aging. In the absence of pathological dementia, however, aging and cognitive decline are not obligatorily linked: some aged individuals exhibit keen learning and memory ability into and beyond their eighth and ninth decades (McClearn, 1997; Backman et al., 2000). In principle, this natural variability could help clarify the mechanisms that underlie age-related cognitive decline.

Like humans, aged rodents exhibit a marked heterogeneity in their ability to learn and remember. Spatial learning is particularly impaired in aged rats, but some aged subjects show severe deficits in spatial learning, whereas others are indistinguishable from young controls (Gage et al., 1984; Barnes and McNaughton, 1985; Deupree et al., 1991; Quirion et al., 1995; Backman et al., 1996). The basis for this variability is unknown, but it is well documented that spatial learning in rodents is strongly hippocampus dependent (Morris et al., 1982, 1990; Hollup et al., 2001). Remarkably, lesions restricted to the dorsal hippocampus or the CA1 cell field have been shown to disrupt spatial learning in rats (Davis et al., 1986; Moser et al., 1995). However, age-related spatial learning deficits in rats do not arise from hippocampal neuron loss (Rapp and Gallagher, 1996), implicating more subtle changes in synaptic structure or function (Rapp et al., 1999; Smith et al., 2000). In line with this thinking, impaired hippocampal synaptic plasticity, specifically long-term potentiation (LTP), has

Received May 23, 2002; revised Aug. 23, 2002; accepted Aug. 29, 2002.

We are grateful to Crista Trippodi for help with the statistical analysis and Heather Bimonte for suggesting the curtain experiment.

Correspondence should be addressed to Dr. Geoffrey C. Tombaugh, Memory Pharmaceuticals Corporation, 100 Philips Parkway, Montvale, NJ 07645. E-mail: tombaugh@memorypharma.com.

Copyright (C) 2002 Society for Neuroscience $\quad 0270-6474 / 02 / 229932-09 \$ 15.00 / 0$ nitude among aged rats was not attributable to an altered response to $5 \mathrm{~Hz}$ stimulation or to differences in the NMDA receptormediated field EPSP. In addition, no performance-related differences in LTP were seen when LTP was induced with 30 or 70 $\mathrm{Hz}$ stimulation protocols. Finally, both $5 \mathrm{~Hz}$ LTP and spatial learning in learning-impaired rats were enhanced with the selective muscarinic $\mathrm{M}_{2}$ antagonist BIBN-99 (5,11-dihydro-8-chloro-11-[[4[3-[(2,2-dimethyl-1-oxopentyl)ethylamino]propyl]-1-piperidinyl] acetyl]-6H-pyrido[2,3-b][1,4]benzodiazepin-6-one). These findings reinforce the idea that distinct types of hippocampal LTP offer mechanistic insight into age-associated cognitive decline.

Key words: hippocampus; aging; spatial learning; LTP; memory; NMDA receptor; muscarinic receptor

been prominently cast as a key factor in age-related cognitive decline (Foster, 1999).

Since its first formal description (Bliss and Lomo, 1973), LTP has been widely, although not unequivocally, implicated in learning and memory (Martin et al., 2000). This link has been strengthened by studies in which hippocampal LTP in aged rats was found to be related to individual differences in spatial learning ability (Barnes and McNaughton, 1985; Deupree et al., 1991; Davis et al., 1993; Bach et al., 1999). Such correlative studies reinforce the idea that the hippocampus plays a key role in spatial memory formation, but whether a selective deficit in LTP per se explains the spatial learning deficit in aged animals is unclear (Shors and Matzel, 1997; McEachern and Shaw, 1999).

Numerous stimulation protocols reliably elicit LTP in the hippocampus. However, the utility of these protocols in probing mnemonic mechanisms may not be equal. Diverse stimulus protocols can trigger increases in synaptic responses [e.g., field EPSPs (fEPSPs)] that are similar in amplitude and stability yet arise from different biochemical cascades depending on the pattern of stimulation, age, species, and even strain of the animal (Shankar et al., 1998; Nguyen et al., 2000; Selcher et al., 2000). If a certain type of LTP does underlie a specific type of learning, then it is logical to imagine a unique set of biochemical mechanisms that both drives LTP expression and is causally linked to the process of information storage. Identifying this type of LTP could help reveal the biochemical intermediates that underlie the learning process.

Any model that attempts to link hippocampal LTP to hippocampal-based learning generates two key predictions. First, LTP deficits should exist only in learning-impaired subjects and should ideally covary with individual learning ability. Second, pharmacological manipulations that enhance learning should also 
augment LTP. In the present study, we describe an in vitro LTP protocol that meets both of these criteria.

\section{MATERIALS AND METHODS}

Animals. Young (Y) (4-6 months old) and aged (24-26 months old) male Fisher 344 rats were obtained from Hilltop Lab Animals (Scottdale, PA). The animals were left undisturbed for a minimum of 1 month in our facility until the beginning of behavioral testing. Rats were housed in pairs in polycarbonate cages $(45 \times 30 \times 18 \mathrm{~cm})$ with corncob bedding and maintained on a $12 \mathrm{hr}$ light/dark schedule (lights off at 7:00 P.M.). Food (LabDiet 5001 rodent diet; Purina Mills, St. Louis, MO) and water were available ad libitum. Animal health was monitored by a veterinarian; animals showing overt signs of morbidity were removed from the study. All procedures were conducted in accordance with local Institutional Animal Care and Use Committee guidelines.

Behavior. Young and aged rats were tested in the Morris water maze as described previously (Rowe et al., 1998). The maze consisted of a 1.6-m-diameter circular pool filled to within $15 \mathrm{~cm}$ of the rim with water $\left(22^{\circ} \mathrm{C}\right)$ made opaque by the addition of nontoxic white latex paint (Createx Colors, East Granby, CT). A circular Plexiglas escape platform (14.5 cm in diameter) was located in the center of one of the quadrants of the pool. The animals were given 15 trials over 5 consecutive days with the platform submerged $2 \mathrm{~cm}$ below the surface of the water (three trials per day; $120 \mathrm{sec}$ maximum trial duration; $20-30 \mathrm{~min}$ intertrial interval). On the last training trial, a probe test was performed in which the retractable Plexiglas platform was pneumatically lowered out of reach of the rats for $30 \mathrm{sec}$ and then returned to its original position for the remainder of the trial. A 40-cm-diameter zone (annulus-40) around the platform center was used to assess probe performance. In some trials, an opaque plastic curtain was placed around the pool perimeter to obscure the extramaze cues. Latencies and swim distances to locate the hidden platform during training trials, time to first entry into and dwell time in the annulus-40, and mean distance from the platform during probe trials were all recorded and analyzed using a computer-based tracking system (San Diego Instruments, San Diego, CA). On day 6, rats not scheduled to receive any additional behavioral testing were given four $60 \mathrm{sec}$ trials in which the platform was raised $2.5 \mathrm{~cm}$ above the water level (visually cued condition) to test for visual, motivational, or motor deficits that may have influenced performance. Rats that required $>40 \mathrm{sec}$ to reach the visible platform on any trial were excluded.

The cognitive status of the aged animals was defined on the basis of their latencies to find the submerged platform on days 3 , 4, and 5 of testing relative to the mean latency of young controls (see Fig. 1). Aged-impaired (AI) rats were defined as those animals whose mean latencies (across the $3 \mathrm{~d}$ of testing) differed by $>3.0$ SDs from that of young controls. Aged animals were considered unimpaired (AU) if their mean latencies were $\leq 0.5 \mathrm{SD}$ from young controls. Aged animals whose mean escape latencies fell between these values were not used in any additional tests.

BIBN-99 (5,11-dihydro-8-chloro-11-[[4-[3-[(2,2-dimethyl-1-oxopentyl) ethylamino]propyl]-1-piperidinyl]acetyl]-6H-pyrido[2,3-b][1,4] benzodiazepin-6-one), a selective muscarinic $\mathrm{M}_{2}$ receptor antagonist (Doods et al., 1993a,b) reported to enhance spatial learning in AI rats (Quirion et al., 1995), was synthesized in our laboratories and stored in a desiccator in powdered form. In behavioral experiments, AI rats treated with BIBN-99 were given an additional $3 \mathrm{~d}$ of training (three trials per day). On each of these days, a $0.5 \mathrm{mg} / \mathrm{kg}$ dose of BIBN-99 (in $0.9 \% \mathrm{NaCl}$, s.c.) or vehicle was administered $45 \mathrm{~min}$ before training began (Quirion et al., 1995). Performance during drug treatment was averaged for the entire $3 \mathrm{~d}$ testing period. On the last day of testing (day 8 , trial 3 ), a $30 \mathrm{sec}$ probe trial was performed on all animals. The visually cued testing procedure (see above) was administered the following day. All statistical analyses, including those for electrophysiological data, were performed either with a $t$ test or a two-way ANOVA. In cases in which the ANOVA detected a significant difference, pairwise comparisons were made (Tukey-Kramer post hoc test) from which $p$ values were derived.

Slice physiology. Rats were anesthetized with isoflurane and killed by decapitation. Transverse hippocampal slices $(400 \mu \mathrm{m})$ were prepared from young adult and a subset of behaviorally characterized aged rats using a tissue chopper. Slices were maintained at $28^{\circ} \mathrm{C}$ in an interface chamber (Fine Science Tools, Foster City, CA) and perfused at 1-2 $\mathrm{ml} / \mathrm{min}$ with artificial CSF (ACSF) that had been preequilibrated with $95 \% \mathrm{O}_{2}-5 \% \mathrm{CO}_{2}$. The ACSF composition was as follows (in $\mathrm{mm}$ ): 124 $\mathrm{NaCl}, 4.5 \mathrm{KCl}, 1 \mathrm{NaH}_{2} \mathrm{PO}_{4}, 26 \mathrm{NaHCO}_{3}, 2.5 \mathrm{CaCl}_{2}, 1.3 \mathrm{MgCl}_{2}$, and 10 glucose. Bipolar stimulating electrodes (stainless steel; Frederick Haer Co., Bowdoinham, ME) and glass recording electrodes (1-3 M $\Omega$; filled with ACSF) were positioned in stratum radiatum of area CA1. Inputoutput curves were recorded, and test stimulus intensity $(0.017 \mathrm{~Hz}, 50$ $\mu$ sec duration) was adjusted to evoke an fEPSP with an initial slope that was $40-45 \%$ of maximum. After a stable baseline period (20-30 min), one of three stimulation protocols was applied at the test intensity: (A) a single $30 \mathrm{sec}$ train at $5 \mathrm{~Hz}$; (B) three $0.5 \mathrm{sec}$ trains at $30 \mathrm{~Hz}(5 \mathrm{~min}$ intertrain interval); or (C) three $0.5 \mathrm{sec}$ trains at $70 \mathrm{~Hz}(5$ min intertrain interval). After the induction protocol, single responses were evoked at the test intensity for 50-60 min (A) or $180 \mathrm{~min}$ (B and C) to monitor LTP amplitude and stability. LTP was measured by comparing the mean fEPSP slope (averaged over $5 \mathrm{~min}$ ) at the end of the post-tetanus period with that of the mean fEPSP slope recorded 5 min before either the tetanus or BIBN-99 application. The effect of NMDA receptor (NMDAR) blockade on $5 \mathrm{~Hz}$ LTP was examined in slices from young rats by exposing the slices to $50 \mu \mathrm{M}$ APV for $15 \mathrm{~min}$ and sequentially applying $5 \mathrm{~Hz}$ stimulation to the same slice, first in the presence of APV and then 60 min later after washout. BIBN-99 was dissolved in DMSO; stock solutions were diluted in ACSF immediately before use and bath applied to slices $30 \mathrm{~min}$ before LTP induction at a final concentration of $1.0 \mu \mathrm{M}$. The DMSO concentration never exceeded $0.05 \%$. NMDA receptor-mediated fEPSPs were isolated by exposing slices for 50-60 min to modified ACSF containing $200 \mu \mathrm{M} \mathrm{MgCl}_{2}, 10 \mu \mathrm{M} \mathrm{CNQX}$, and 10 $\mu \mathrm{M}$ glycine.

Slices from aged rats subjected to 5 or $30 \mathrm{~Hz}$ stimulation were prepared from different hippocampi of the same rat; experimenters were blind to the behavioral status of the animal. Slices subjected to $70 \mathrm{~Hz}$ stimulation were taken from a separate group of rats. In all cases, recordings from young and aged slices were interleaved. Recordings on a given day were typically made from multiple slices (two to four) from each animal. For LTP behavior correlations and NMDAR fEPSP measures, the datum for a given animal was represented by the average value from all slices examined. In experiments in which BIBN-99 was applied, paired control recordings were always performed in adjacent slices from the same animal. Complex spiking observed during $5 \mathrm{~Hz}$ stimulation was quantified by counting the number of negative spikes clearly resolved from the fEPSP (Thomas et al., 1998). Slices that did not exhibit stable fEPSP baselines, or those in which the presynaptic fiber volley changed abruptly or shifted by $>20 \%$ during the course of the experiment, were excluded from analysis. Input-output curves are plotted as fEPSP slope versus fiber volley amplitude. All data are presented as mean \pm SEM. Sample sizes are given as the number of slices, with the number of animals shown in parentheses.

\section{RESULTS}

\section{Learning impairments can be observed in a subset of aged rats}

Aged rats were characterized as either AI or AU based on their mean escape latency derived from training days 3-5 in the Morris water maze. As anticipated from previous studies, aged rats showed significantly longer swim times and swim distances with a greatly increased variance compared with young controls. Aged rats swam more slowly than young rats (young, $27.9 \pm 0.6 \mathrm{~cm} / \mathrm{sec}$; AU, $24.9 \pm 0.4 \mathrm{~cm} / \mathrm{sec}$; AI, $23.1 \pm 0.5 \mathrm{~cm} / \mathrm{sec})$. Importantly, there was no significant difference in swim speed between AU and AI animals. Swim times and distances were highly correlated for all groups ( $r>0.9$ in all cases).

Swim times for aged rats exhibited a skewed, unimodal distribution (Fig. 1A). Of 89 aged animals screened in the water maze for the present study, $40(45 \%)$ were classified as AI, and 21 (24\%) were classified as AU (see Materials and Methods). The remainder of the animals did not fall clearly into either category and were excluded from additional study. The acquisition curves for the AU and AI groups illustrate the dramatic impairment in the ability of AI animals to find the hidden platform (Fig. $1 B, C$ ). However, when the platform was made visible, all young and AU and all but four AI rats were able to achieve criterion performance (see Materials and Methods). The mean swim times for young, AU, and AI rats that reached criterion in the visually cued 

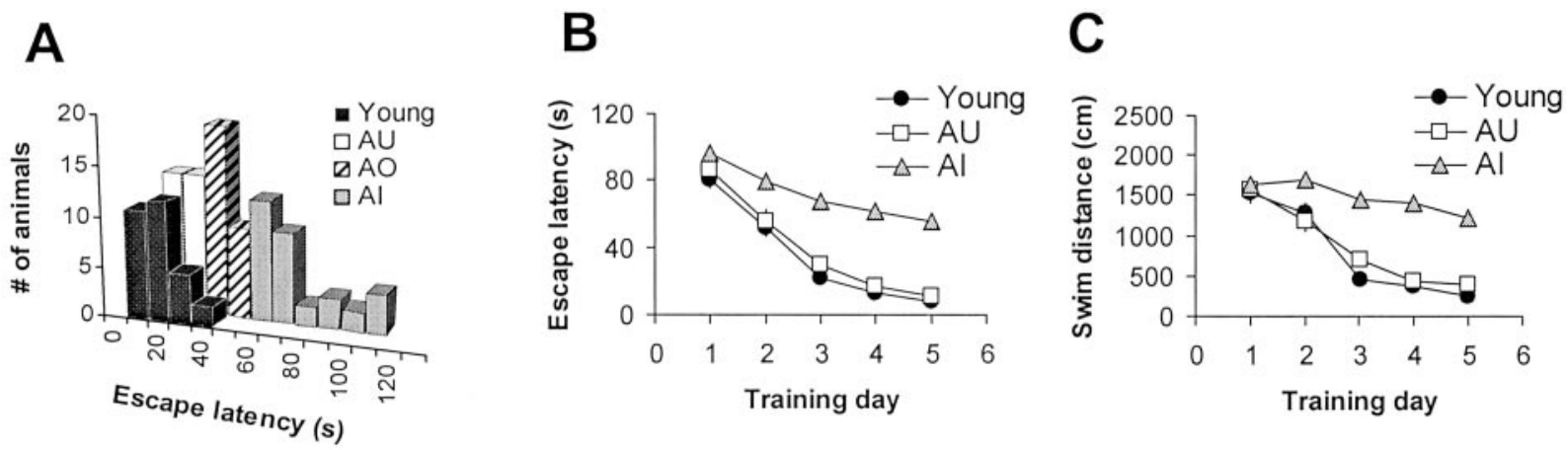

Figure 1. Morris water maze testing reveals a broad gradient of learning ability among aged F344 rats. $A$, Mean escape latencies measured on training days 3-5 were grouped in $10 \mathrm{sec}$ bins for both aged (24-26 months) and young (4-6 months) rats. AU or AI animals were identified as those whose mean performance differed by $<0.5$ or $>3$ SDs from the mean of young controls, respectively. The remaining rats [aged-other $(A O)$ ] were excluded from additional study. The acquisition curves during training are plotted for both mean escape latency $(B)$ and path length $(C)$. Error bars in $B$ and $C$, when not visible, are smaller than the symbol size.
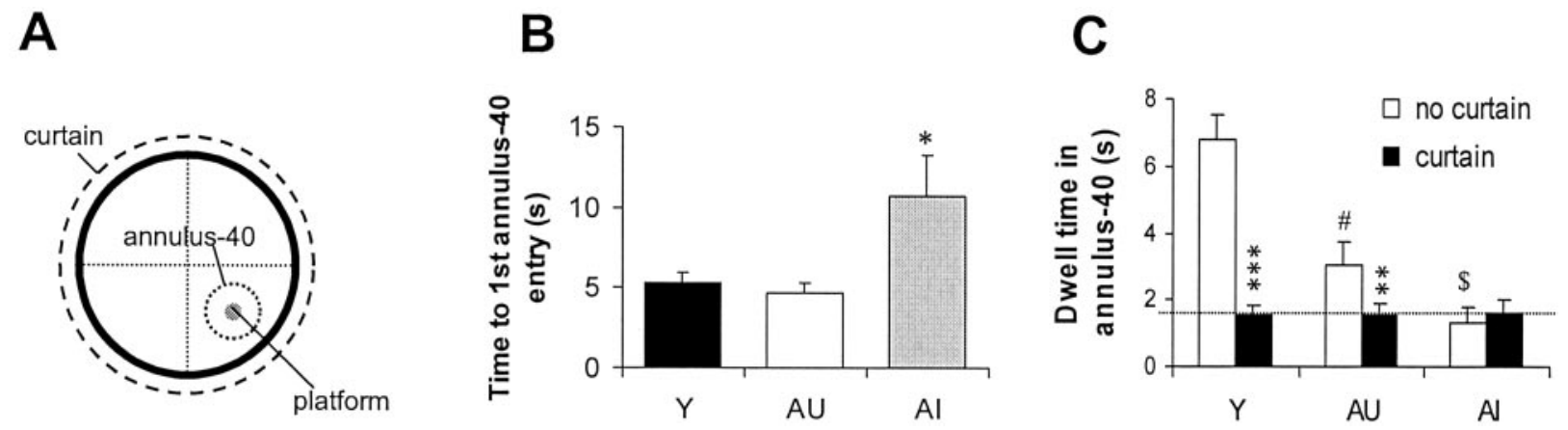

Figure 2. Probe measures indicate that AI rats do not use a spatial strategy in the water maze. $A$, Schematic of the water maze. $B$, With the platform removed, AI rats required significantly more time for their first entry into the annulus-40 than AU or young rats. AI and AU data were derived from animals used for subsequent electrophysiological analysis in Figures 4 and 5 (Y, $n=36$; AU, $n=7$; AI, $n=13$ ). ${ }^{*} p<0.05$ compared with AU group. $C$, AI rats spent less time within the target area than AU or Y rats. Dwell time for AI rats (dashed line), unlike that for AU and Y rats, was unchanged when the extramaze cues were obscured by a curtain (Y, $n=12$; AU, $n=11$; AI, $n=11$ ). ${ }^{* *} p<0.01$ and ${ }^{* * *} p<0.001$ compared with matching "no curtain" control (paired $t$ test). \#p $<0.05$ and $\$ p<0.05$ compared with Y and AU no curtain groups, respectively (unpaired $t$ test).

trials were $5.0 \pm 0.3,8.5 \pm 1.0$, and $8.8 \pm 0.8 \mathrm{sec}$, respectively. These data suggest that the altered ability of the AI animals to locate the submerged platform was not attributable to a visual, motor, or motivational deficit.

\section{Al rats do not use a spatial strategy in the water maze}

At the end of the $5 \mathrm{~d}$ training period, each animal was given a 30 sec probe test, during which the submerged escape platform was unavailable. The swim speeds of AU and AI rats did not differ during the probe trial $(\mathrm{AU}, 23.9 \pm 0.6 \mathrm{~cm} / \mathrm{sec}$; AI, $23.4 \pm 0.4$ $\mathrm{cm} / \mathrm{sec})$ and were comparable with that of young controls $(25.4 \pm$ $0.5 \mathrm{~cm} / \mathrm{sec})$. However, AI animals required significantly more time to make their first entry into the annulus-40 compared with either AU or young rats ( $p<0.05$; ANOVA) (Fig. $2 A)$. The AI rats also maintained a significantly greater mean distance from the platform during the probe trial ( $p<0.05$; ANOVA; data not shown). Young and AU rats had nearly identical first entry latencies (Fig. 2B).

Consistent with the first-entry data, AI rats spent significantly less time $(1.32 \pm 0.5 \mathrm{sec})$ swimming in the annulus-40 than either $\mathrm{AU}(3.1 \pm 0.7 \mathrm{sec} ; p<0.05)$ or young $(6.8 \pm 0.7 \mathrm{sec} ; p<0.001)$ rats. Surprisingly, the dwell time for AU rats was also significantly smaller than young controls ( $p<0.01$; ANOVA). This finding suggested that AU rats may have used a distinct (i.e., nonspatial) strategy to locate the platform and prompted us to examine this question in more detail. New groups of identically trained animals were subjected to a revised probe analysis in which a curtain was placed around the pool to obscure the extramaze cues (Fig. 2C). No-curtain dwell times in all groups were nearly identical to those seen in the first study. With the curtain present, however, dwell times for young and AU rats fell to the level seen in AI rats, whereas the dwell time for the AI rats was unchanged.

\section{BIBN-99 improves spatial learning in Al rats}

Based on previous studies (Quirion et al., 1995), we chose to use BIBN-99 in the current study to probe the linkage between spatial learning and LTP in AI rats. As shown previously, AI rats treated with BIBN-99 $1 \mathrm{hr}$ before retesting in the water maze task exhibited significantly shorter escape latencies than the vehicle-treated controls ( $p<0.01$; paired $t$ test) (Fig. 3A). Treatment with BIBN-99 did not significantly alter swim speed (vehicle, $20.3 \pm 0.5$ $\mathrm{cm} / \mathrm{sec}$; BIBN-99, $20.4 \pm 0.6 \mathrm{~cm} / \mathrm{sec})$. During the probe trial, latency to first annulus-40 entry was significantly reduced, whereas dwell time within the annulus-40 was significantly increased in the drug-treated group ( $p<0.01$; paired $t$ test) (Fig. 3B,C).

\section{Slices from young and aged rats respond similarly to electrical stimulation}

Input-output (stimulus-response) curves were generated from slices from Y AU and AI rats by plotting the fEPSP slope as a 

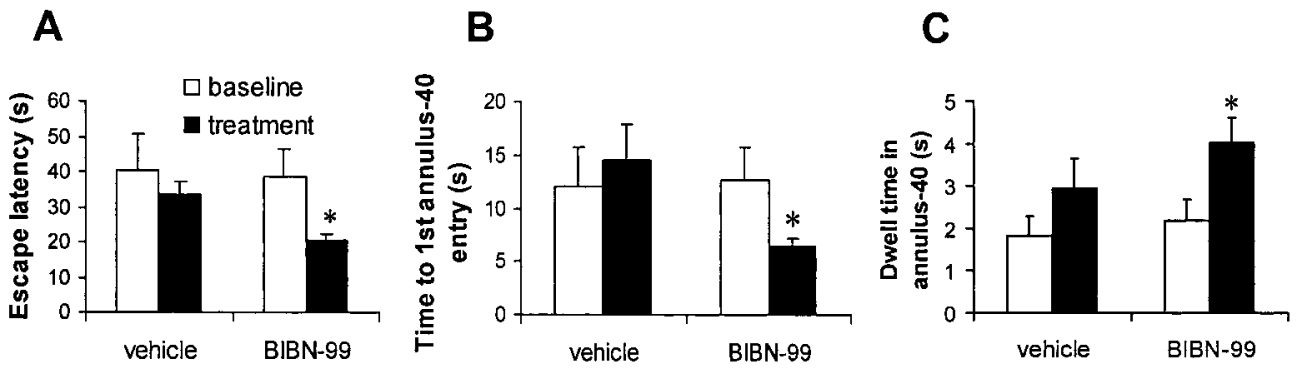

Figure 3. BIBN-99 improves water maze performance in AI rats. The mean escape latency $(A)$ and latency to first entry into the annulus-40 $(B)$ were reduced, whereas dwell time in the annulus- $40(C)$ was significantly increased after a single injection of BIBN-99 given 45 min before each set of training trails over $3 \mathrm{~d}$. Baseline data refer to those collected on training days $3-5$ for rats that received either vehicle $(n=8)$ or BIBN-99 $(n=11)$ injections on days $6-8 .{ }^{*} p<0.01$ compared with pretreatment baseline (paired $t$ test).

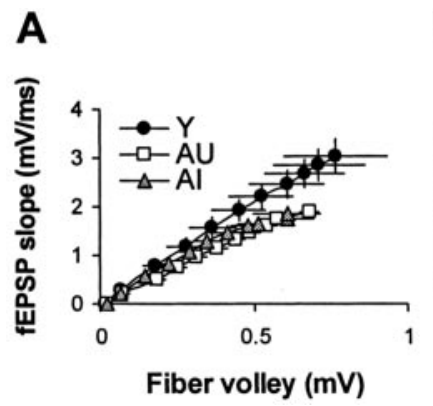

B

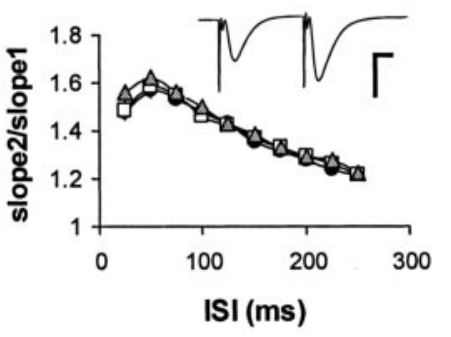

Figure 4. Slices from AU and AI rats exhibit no differences in CA1 synaptic transmission and short-term facilitation. $A$, Input-output curves reveal depressed synaptic transmission for aged rats relative to young controls but were not different between AU and AI subgroups [Y, $n=$ 9(7); AU, $n=9(7)$; AI, $n=19(8)]$. $B$, Paired-pulse facilitation in CA1 was indistinguishable between young, $\mathrm{AU}$, and $\mathrm{AI}$ groups across a range of interstimulus intervals (ISI). Inset shows a representative trace (young rat). Calibration: $2 \mathrm{mV}, 10 \mathrm{msec}$.

function of fiber volley amplitude. For a given fiber volley amplitude, evoked responses from aged slices were consistently smaller than those from young controls (Fig. 4A). However, input-output curves for AU and AI animals were not statistically different. In addition, no significant difference in paired-pulse facilitation was observed among the groups across a range of interstimulus intervals (Fig. 4B).

\section{Theta-frequency stimulation in vitro reveals an LTP deficit in Al rats}

Thirty seconds of $5 \mathrm{~Hz}$ stimulation routinely induced robust LTP in slices taken from young rats. This form of potentiation rose gradually and stabilized within $10-15$ min after the $5 \mathrm{~Hz}$ train (Fig. 5A). LTP in slices from AU rats was comparable with that seen in young animals, but LTP elicited from AI slices was significantly smaller ( $p<0.001$; ANOVA) (Fig. $5 A)$. During $5 \mathrm{~Hz}$ stimulation, complex spiking gradually emerged and was observed in all three groups (Fig. 5B). No dramatic differences were seen in the average latency to spiking, as measured by the stimulus number at which one-half of the slices experienced the first spike (Y, 54; AU, 50; AI, 53). We also found no difference in the total number of spikes evoked $(\mathrm{Y}, 131 \pm 10$; $\mathrm{AU}, 120 \pm 11$; AI, $131 \pm 18)$.

To determine whether the depressed LTP observed in AI rats was a function of the $5 \mathrm{~Hz}$ stimulation protocol, parallel experiments were run in which higher-frequency stimulation was applied to slices taken from the opposite hippocampus of the same animals. In these slices, LTP was induced with three $0.5 \mathrm{sec}$ trains of $30 \mathrm{~Hz}$ stimulation, with a 5 min intertrain interval (Fig. $5 C$ ). Under these conditions, LTP amplitude at $3 \mathrm{hr}$ was significantly reduced in both $\mathrm{AU}$ and $\mathrm{AI}$ rats $(p<0.05)$ compared with young controls, but no statistically significant AU-AI difference emerged. In slices from a separate set of animals, increasing the stimulation frequency to $70 \mathrm{~Hz}$ generated a more robust but equivalent level of LTP in all three groups (Fig. 5D).

\section{LTP deficits in Al rats do not arise from reduced NMDA receptor function}

LTP at $5 \mathrm{~Hz}$ in adult mice has been shown previously to be partially NMDA receptor dependent (Thomas et al., 1998). In agreement with this finding, bath application of $50 \mu \mathrm{M}$ APV significantly depressed, but did not completely block, $5 \mathrm{~Hz}$ LTP in young adult rats (Fig. 6A). Exposure to APV also completely prevented complex spiking during $5 \mathrm{~Hz}$ stimulation. In these experiments, a second train of $5 \mathrm{~Hz}$ stimulation applied in the same slice 50 min after APV washout resulted in complex spiking (total spike number, $98 \pm 12$ ) and robust LTP [158 $\pm 6 \% ; n=$ $7(4)]$ that was comparable with that seen in naïve young slices $[162 \pm 7 \% ; n=17(8)]$. Because NMDAR subunit expression is depressed in aged rats (Davis et al., 1993; Adams et al., 2001), we considered the possibility that the selective impairment in $5 \mathrm{~Hz}$ LTP in AI rats reflected a reduced capacity for LTP induction. To address this question directly, we examined NMDAR-mediated responses in young, $\mathrm{AU}$, and $\mathrm{AI}$ rats. In the presence of CNQX, glycine, and low $\mathrm{Mg}^{2+}$, fEPSPs evoked in all groups could be blocked with $50 \mu \mathrm{M}$ APV (data not shown). NMDA receptormediated fEPSPs recorded from both AU and AI slices were smaller on average $(\sim 30 \%)$ than those seen in young controls over a range of stimulus intensities (Fig. 6B). However, the NMDA fEPSPs recorded in slices from AI and AU animals were indistinguishable from each other.

\section{LTP at $5 \mathrm{~Hz}$ correlates with performance in the Morris water maze}

To further explore the possible link between $5 \mathrm{~Hz} \mathrm{LTP}$ and spatial learning, we plotted LTP amplitude, measured at $1 \mathrm{hr}$ after LTP induction, against three behavioral measures (Fig. 7). Regression analysis revealed a strong negative correlation between 5 $\mathrm{Hz}$ LTP and mean escape latency among aged rats $(p<0.001)$ (Fig. $7 A$ ). LTP at $5 \mathrm{~Hz}$ was also highly correlated with two measures of probe trial performance: (1) latency to the first entry into the annulus-40 $(p<0.05)$ (Fig. $7 B)$; and (2) cumulative dwell time in the annulus-40 $(p<0.01)$ (Fig. $7 C)$. 

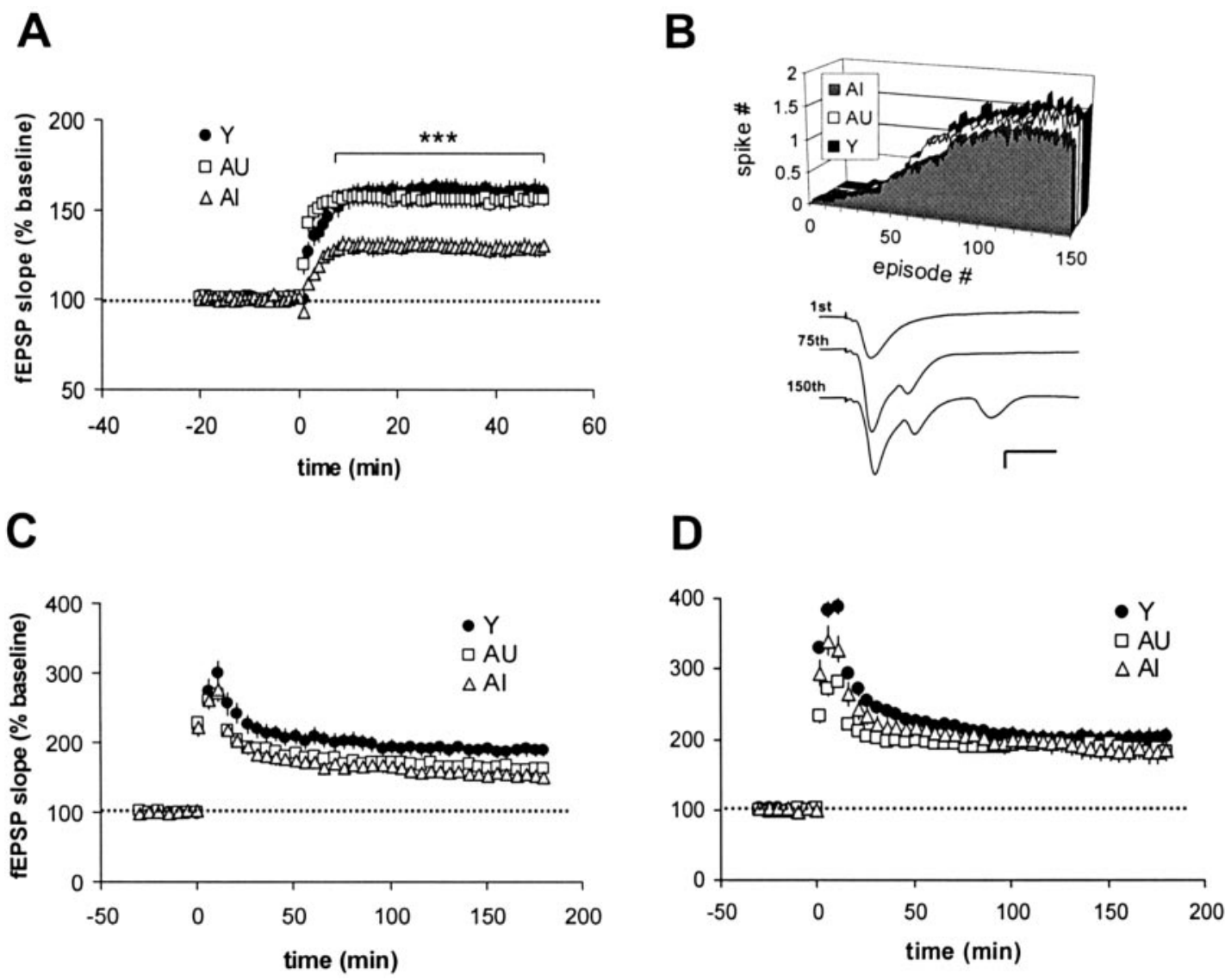

Figure 5. LTP at $5 \mathrm{~Hz}$ distinguishes between slices from AI and AU rats. $A$, AI slices subjected to $5 \mathrm{~Hz}, 30$ sec stimulation exhibited significantly smaller synaptic potentiation in CA1 than that seen in either young or AU slices [Y, $n=17(8)$; AU, $n=20(7)$; AI, $n=32(13)]$. *** $p<0.001$ compared with young and AU. $B$, All groups responded to $5 \mathrm{~Hz}$ stimulation with a similar time course and number of complex spikes. Inset in $B$ shows a representative set of traces collected during $30 \mathrm{sec}$ of $5 \mathrm{~Hz}$ stimulation in a young slice. Calibration: $1 \mathrm{mV}, 10 \mathrm{msec}$. $C$, Slices taken from the contralateral hippocampus of a subset of animals in $A$ were subjected to three $0.5 \mathrm{sec}$ trains at $30 \mathrm{~Hz}$ stimulation. The resulting LTP in both AU and AI slices was smaller than that in young controls $(p<0.05)$, but no AU-AI difference was detected [Y, $n=17(14)$; AU, $n=11(6)$; AI, $n=8(5)]$. $D$, Stimulation at 70 Hz in a separate set of rats induced robust, long-lasting, and statistically equivalent LTP in all groups [Y, $n=13(11) ; \mathrm{AU}, n=17(9)$; AI, $n=8(7)]$. Data points in $C$ and $D$ are presented at $5 \mathrm{~min}$ intervals for clarity.

\section{BIBN-99 enhances 5 Hz LTP}

Given that BIBN-99 improved water maze performance in AI rats, we tested the possible link between $5 \mathrm{~Hz}$ LTP and spatial learning by asking whether BIBN-99 could also enhance $5 \mathrm{~Hz}$ LTP. Bath application of $1 \mu \mathrm{M}$ BIBN-99 to AI slices for $30 \mathrm{~min}$ had no observable effects on baseline responses either during drug delivery or $60 \mathrm{~min}$ after washout $[104 \pm 4 \% ; n=5(2)]$. In contrast, BIBN-99 significantly enhanced the degree of synaptic potentiation in AI slices after $5 \mathrm{~Hz}$ stimulation $(p<0.001)$ (Fig. $8 A$ ). This action was probably unrelated to any direct potentiating effect on the NMDA receptor itself, because a 30 min application of $1 \mu \mathrm{M}$ BIBN-99 did not alter the slope of the isolated NMDA fEPSP recorded in AI slices $(103 \pm 2 \%$ of baseline; $n=$ $6)$. In addition, BIBN-99 did not change the onset or total number of complex spikes evoked during the $5 \mathrm{~Hz}$ train (Fig. $8 B$ ). In contrast to its effect on $5 \mathrm{~Hz}$ LTP, BIBN-99 $(1 \mu \mathrm{M})$ failed to enhance LTP in AI slices evoked by repeated $70 \mathrm{~Hz}$ trains (Fig. $8 C$ ). Finally, the same concentration of BIBN-99 did not modify $5 \mathrm{~Hz}$ LTP recorded in slices from young rats (control, $152 \pm 8 \%$; BIBN-99, $144 \pm 7 \%$ ).

\section{DISCUSSION}

The key findings in this study are that a specific form of hippocampal LTP in aged rats evoked by theta-frequency stimulation
(1) strongly correlates with individual performance on a spatial learning task and (2) can be amplified by a selective muscarinic $\mathrm{M}_{2}$ antagonist that also improves spatial learning.

One critical element in this study that enabled us to examine the linkage between LTP and learning was our ability to distinguish learning-impaired from unimpaired aged rats. Although our definition of "impaired" is statistically based and not absolute, this approach helped create more uniform experimental groups and removed aging per se as a variable. Compared with young rats, aged rats exhibited a right-shifted, broadly skewed gradient of water maze performance (escape latency). This distribution appeared unimodal, suggesting that both AU and AI animals were members of the same population, as might be expected for the inbred Fischer 344 (F344) strain. Thus, agerelated cognitive decline in the rat, although often observed, is not an inevitable consequence of aging.

After the $5 \mathrm{~d}$ training period, probe trial analysis showed that both young and AU rats entered the annulus- 40 with the same latency, suggesting an equivalent certainty of the platform location. Surprisingly, the AU rats spent significantly less time in the annulus-40 with fewer platform crossings (data not shown) than young rats during the probe trial. This difference in perseverance suggests that AU rats either (1) recognized that the platform was absent and adapted by deliberately searching elsewhere or (2) 
A

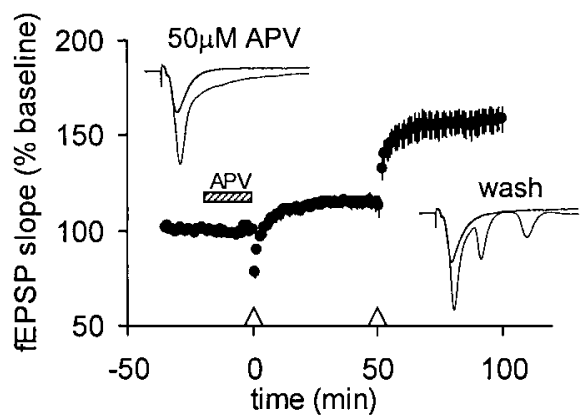

B

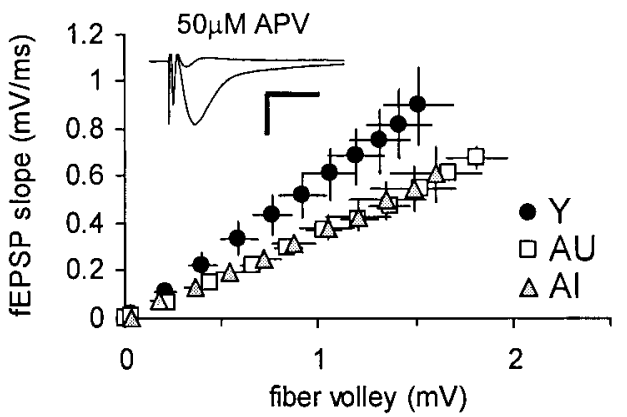

Figure 6. The deficit in $5 \mathrm{~Hz}$ LTP in AI rats is not linked to a loss of NMDA receptor function. $A$, LTP at $5 \mathrm{~Hz}$ in young slices $[n=7(4)]$ is partially NMDA receptor dependent, because bath application of $50 \mu \mathrm{M}$ APV (hatched bar) depressed but did not completely block LTP triggered by $5 \mathrm{~Hz}, 30 \mathrm{sec}$ stimulation $(\Delta)$. Insets depict the 1 st and 150th sweeps recorded from the same slice during the two successive $5 \mathrm{~Hz}$ trains. Complex spiking during $5 \mathrm{~Hz}$ stimulation in the presence of APV was abolished. After washout, $5 \mathrm{~Hz}$ stimulation triggered complex spiking (total spikes, $98 \pm 12$ ) and robust LTP that was comparable with

that typically seen in naïve control slices (see Fig. 5). $B$, The NMDA receptor-mediated fEPSP, isolated in the presence of $10 \mu \mathrm{M} C N Q X, 10 \mu \mathrm{M}$ glycine, and $0.2 \mathrm{mM} \mathrm{Mg}^{2+}$ was depressed in both $\mathrm{AU}$ and $\mathrm{AI}$ rats by $\sim 30 \%$ compared with young controls across a range of stimulus intensities [Y, $n=21(7)$; AU, $n=18(6)$; AI, $n=13(6)]$. When normalized to fiber volley amplitudes $>0.5 \mathrm{mV}$, slope values for both AU and AI rats were significantly different from young controls $(p<0.01)$. No difference between AU and AI rats was detected. Inset depicts representative traces (average of 5 sweeps) recorded from an aged rat slice before and after exposure to $50 \mu \mathrm{M}$ APV. Calibration: $1 \mathrm{mV}, 10 \mathrm{msec}$.

A

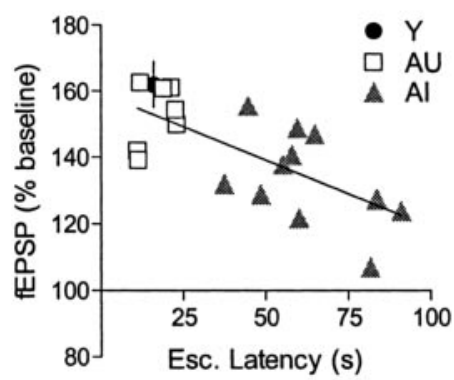

B

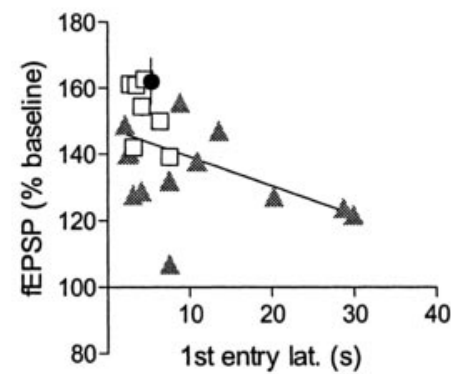

C

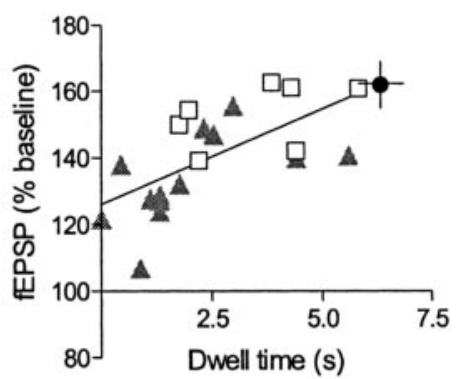

Figure 7. LTP at $5 \mathrm{~Hz}$ correlates with water maze learning in aged rats. $A$, The average level of $5 \mathrm{~Hz} \mathrm{LTP}$ calculated for each aged animal was strongly correlated to individual mean escape latency in the water maze $\left(A ; R^{2}=0.50 ; p<0.001\right)$. In addition, $5 \mathrm{~Hz}$ LTP was significantly correlated to two probe measures: the latency to first entry into the annulus- $40\left(B ; R^{2}=0.24 ; p<0.05\right)$ and total dwell time in the annulus-40 $\left(C ; R^{2}=0.42 ; p<0.01\right)$. In each case, the data for young control rats are presented for comparative purposes only and were not included in the regression analysis.

grew less certain about the platform location and swam randomly. We are currently unable to distinguish between these two possibilities. Although AU and Y rats perform similarly in the water maze, they are not identical in all measures and thus cannot be viewed as equivalent. However, the critical fact remains that both $\mathrm{Y}$ and AU rats were clearly dependent on extramaze cues and thus, unlike AI rats, used a spatial (i.e., hippocampus dependent) strategy to solve the task.

Changes in hippocampal synaptic function have been associated previously with age-related memory loss (Barnes, 1994; Foster and Norris, 1997). We found that both baseline CA3-CA1 synaptic transmission and NMDA receptor-mediated responses were blunted in aged rats, as reported previously (Landfield et al., 1986; Deupree et al., 1993; Barnes et al., 1997; Norris et al., 1998b; Eckles-Smith et al., 2000). However, neither measure differed between AU and AI subjects. Our NMDA fEPSP data are consistent with reduced NMDA receptor expression in the aged rat brain (Eckles-Smith et al., 2000; Clayton and Browning, 2001), but this reduction appears not to underlie the behavioral and LTP differences between AU and AI rats that we observed.

Hippocampal NMDAR expression is required for normal spatial learning in young rats and mice (Tsien et al., 1996; Clayton et al., 2002), but the cognitive impact of a modest decline in NMDA receptor number in aged rats remains unclear. Studies of the relationship between hippocampal NMDA receptor expression and water maze performance among aged rats have yielded mixed results (Clark et al., 1992; Davis et al., 1993; Adams et al., 2001; Magnusson, 2001). NMDA receptors in the aged rat brain function normally, and CA1 neurons from aged rats can express robust NMDAR-dependent LTP that is indistinguishable from young controls (Deupree et al., 1993; Barnes et al., 1996; Billard et al., 1997). In fact, age-related impairments in CA1 LTP are generally obscured by high-frequency stimulation but are revealed when weaker, near-threshold stimulus protocols are applied (Deupree et al., 1993; Moore et al., 1993; Norris et al., 1996). These data collectively support the conclusion that fewer functional excitatory CA1 synapses exist in aged rats (Barnes et al., 1992, 1997), although it is not known whether this synapse loss covaries with learning ability.

Our in vitro experiments highlight the ability of $5 \mathrm{~Hz}$, but not 30 or $70 \mathrm{~Hz}$, stimulation to generate a form of LTP that distinguishes AU from AI rats. A general assumption we made is that a change in synaptic efficacy is required for hippocampal-based learning and that these two processes are driven by an overlapping set of cellular mechanisms. A unique consequence of orthodromic theta-frequency stimulation in CA1 is the gradual induction of synchronized complex burst firing in the pyramidal cells. These complex spikes mimic the firing pattern of CA1 neurons in vivo (Kandel and Spencer, 1961; Suzuki and Smith, 1985; Thomas et al., 1998). Moreover, burst firing in CA1 occurs at theta frequencies $(5-12 \mathrm{~Hz})$ during exploratory behavior (Otto et al., 1991) and is required for $5 \mathrm{~Hz}$ LTP induction in vitro (Thomas et 


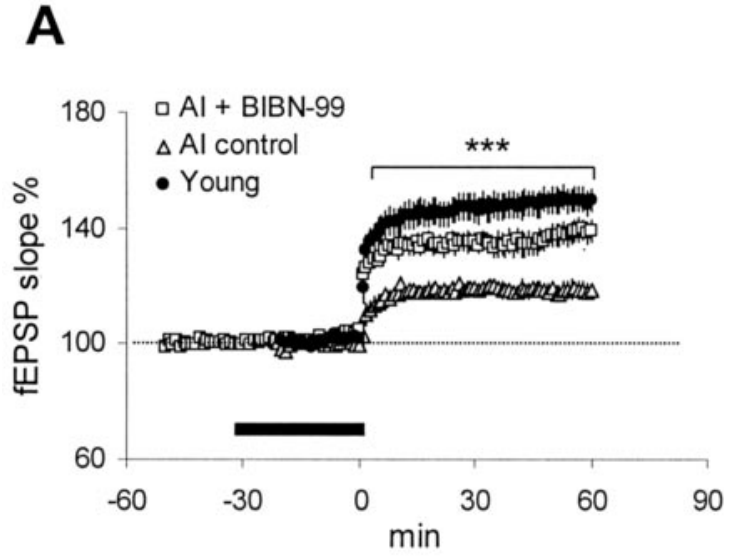

B
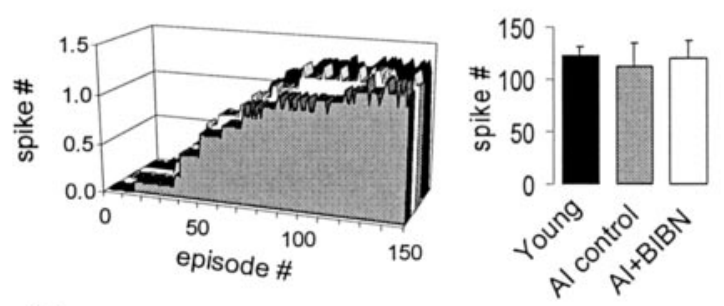

C

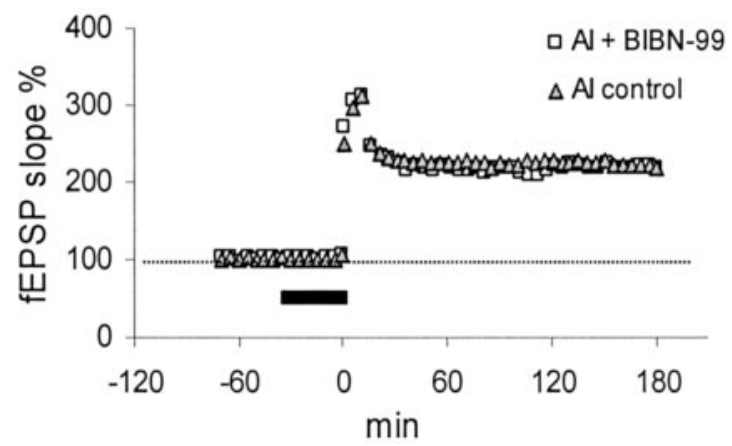

Figure 8. BIBN-99 enhances $5 \mathrm{~Hz}$ LTP in slices from AI rats. A, Bath application of $1 \mu \mathrm{M}$ BIBN-99 (thick bar) significantly enhanced $5 \mathrm{~Hz}$ LTP in AI slices $[n=9(5)]$ to a level slightly smaller than that seen in young controls $[n=17(8)]$. ${ }^{* * *} p<0.001$, AI plus BIBN-99 compared with AI control. $B$, BIBN-99 did not significantly affect the time course or total number of complex spikes evoked during $5 \mathrm{~Hz}$ stimulation. $C$, BIBN-99 had no effect on LTP evoked by repeated trains of $70 \mathrm{~Hz}$ stimulation in AI slices (see Materials and Methods). Data points in $C$ are presented at 5 min intervals for clarity. Error bars were smaller than the symbol size.

al., 1998; Pike et al., 1999; Paulsen and Sejnowski, 2000). We find that complex spiking is inhibited by NMDAR blockade, consistent with previous findings in vivo (Abraham and Kairiss, 1988) but in sharp contrast to a recent study in which $5 \mathrm{~Hz}$ spiking in adult mouse slices was reportedly unaffected by D-APV (Thomas et al., 1996). The basis for this apparent species difference is unclear.

Putative mechanisms for theta-frequency LTP have been described previously (Blitzer et al., 1995, 1998; Thomas et al., 1996; Winder et al., 1999), but the basis for the AU-AI difference in 5 Hz LTP that we report is not known. One possible scenario could involve a difference in calcium channel signaling. L-type calcium channels in rat CA1 neurons increase with age (Landfield et al., 1989; Campbell et al., 1996) and have been linked to the slow afterhyperpolarization (sAHP) (Marrion and Tavalin, 1998; Bowden et al., 2001). CA1 neurons in aged rodents exhibit both a larger sAHP and depressed membrane excitability that vary inversely with hippocampal-dependent learning (Landfield and Pitler, 1984; Moyer et al., 1992; Disterhoft et al., 1996). Because the sAHP conductance has been mapped to the proximal apical dendrites (Sah and Bekkers, 1996) and peaks 200-400 msec after a spike burst, a larger sAHP could blunt $5 \mathrm{~Hz}$ LTP by impeding action potential propagation into the dendrites, in which spikes presumably amplify $\mathrm{Ca}^{2+}$ influx. Notably, L-type calcium channel blockers facilitate hippocampal (CA1) $5 \mathrm{~Hz}$ LTP in aged but not in young adult F344 rats (Norris et al., 1998a) and fail to affect $5 \mathrm{~Hz}$ LTP in young mice (Thomas et al., 1998).

We found that BIBN-99 enhanced spatial learning in AI F344 rats, confirming and extending the results of previous studies that used other rat strains (Pike and Hamm, 1995; Quirion et al., 1995). The additional value of this result is that it provided a tool to test the relationship between LTP and spatial learning. An important criterion of any cellular mechanism thought to govern a complex behavior is that the mechanism should be sensitive to agents capable of enhancing that behavior. Our experiments with BIBN-99 fulfill this criterion and strengthen the link between 5 Hz LTP and spatial learning, suggesting that the two phenomena share a common pathway.

How might $\mathrm{M}_{2}$ blockade influence $5 \mathrm{~Hz}$ LTP? $\mathrm{M}_{2}$ receptors in the CNS are thought to exist primarily, although not exclusively, as inhibitory presynaptic autoreceptors (Levey et al., 1991; Bogdanovic et al., 1993; Auerbach and Segal, 1996; Kitaichi et al., 1999). The efficacy of BIBN-99 in aged but not young tissue implies that $\mathrm{ACh}$, released either tonically or during theta stimulation, is a limiting factor for $5 \mathrm{~Hz}$ LTP induction in AI rats. In this regard, basal hippocampal ACh levels in situ are not only reduced in aged F344 rats, but both BIBN-99 and the structurally related $\mathrm{M}_{2}$ blocker AFDX384 ( \pm )-5,11-dihydro-11-([2-[2-[(dipropylamino) methyl]-1-piperidynyl)ethyl)amino]carbonyl)-6H-pyrido[2,3b] $(1,4)$-benzodiazepine-6-one) have been shown to elevate hippocampal ACh levels more robustly in aged than young subjects (Quirion et al., 1995; Vannucchi et al., 1997). Blockade of $\mathbf{M}_{2}$ receptors on ACh terminals could facilitate ACh release and enhance pyramidal cell excitability via diverse postsynaptic mechanisms, including inhibition of the M-current and the sAHP (Brown and Adams, 1980; Pitler and Alger, 1990; Krause and Pedarzani, 2000; Krause et al., 2002). Such actions could enhance complex spike probability (Azouz et al., 1996) and spike propagation into the dendrites (Tsubokawa and Ross, 1997), which is critical to the induction of $5 \mathrm{~Hz}$ LTP.

If the relevant consequence of $\mathrm{M}_{2}$ blockade in our study was to elevate synaptic ACh levels, cholinesterase blockade should also enhance $5 \mathrm{~Hz}$ LTP. However, we found that the clinically prescribed AChE inhibitor donepezil (Aricept; Eisai, Tokyo, Japan) had no effect on $5 \mathrm{~Hz}$ LTP in AI slices when applied at either 1 or $10 \mu \mathrm{M}$ (data not shown). This negative finding is not likely attributable to the absence of AChE itself (Zhang et al., 1997) but could reflect an ambient ACh level too low for AChE inhibition to have had any meaningful effect. Conversely, BIBN-99 could have enhanced $5 \mathrm{~Hz}$ LTP via a different route.

The vast majority of $\mathrm{M}_{2}$ receptors in the hippocampus reside as heteroreceptors on GABAergic terminals (Rouse et al., 2000). Some of these terminals arise either from septal GABA-containing afferents or intrinsic "disinhibitory" GABAergic cells, both of 
which impinge on other "inhibitory" interneurons (Freund and Antal, 1988; Gulyas et al., 1996; Hajos et al., 1998), which in turn exert powerful inhibitory control over large numbers of principal cells. These anatomical data are consistent with the disinhibitory action of septal stimulation on hippocampal pyramidal neurons (Toth et al., 1997), and $\mathrm{M}_{2}$ blockade may facilitate this disinhibition by promoting GABA release onto inhibitory cells. During $5 \mathrm{~Hz}$ stimulation, disinhibition of pyramidal cells could promote more extensive spike backpropagation (Tsubokawa and Ross, 1996) and thus more robust $\mathrm{Ca}^{2+}$ signaling.

We chose to examine BIBN-99 in our LTP paradigm because it provided a useful tool to test the putative linkage between spatial learning and LTP. Although muscarinic agents are commonly used to investigate cognitive mechanisms, our results with BIBN-99 do not necessarily suggest a privileged role for cholinergic mechanisms in learning and memory. In fact, ongoing studies in our laboratory have shown that $\mathrm{M}_{2}$ antagonism is not the only route by which $5 \mathrm{~Hz}$ LTP and spatial learning can be enhanced. Identifying the key biochemical pathways relevant to both LTP and mnemonic processes remains a large challenge. Our findings illustrate how one can exploit LTP as a tool for identifying learning-memory mechanisms without requiring a detailed understanding of LTP itself.

\section{REFERENCES}

Abraham WC, Kairiss EW (1988) Effects of the NMDA antagonist 2AP5 on complex spike discharge by hippocampal pyramidal cells. Neurosci Lett 89:36-42.

Adams MM, Smith TD, Moga D, Gallagher M, Wang Y, Wolfe BB, Rapp PR, Morrison JH (2001) Hippocampal dependent learning ability correlates with $N$-methyl-D-aspartate (NMDA) receptor levels in CA3 neurons of young and aged rats. J Comp Neurol 432:230-243.

Auerbach JM, Segal M (1996) Muscarinic receptors mediating depression and long-term potentiation in rat hippocampus. J Physiol (Lond) 492:479-493.

Azouz R, Jensen MS, Yaari Y (1996) Ionic basis of spike afterdepolarization and burst generation in adult rat hippocampal CA1 pyramidal cells. J Physiol (Lond) 492:211-223.

Bach ME, Barad M, Son H, Zhuo M, Lu YF, Shih R, Mansuy I, Hawkins RD, Kandel ER (1999) Age-related defects in spatial memory are correlated with defects in the late phase of hippocampal long-term potentiation in vitro and are attenuated by drugs that enhance the cAMP signaling pathway. Proc Natl Acad Sci USA 96:5280-5285.

Backman C, Rose GM, Hoffer BJ, Henry MA, Bartus RT, Friden P, Granholm AC (1996) Systemic administration of a nerve growth factor conjugate reverses age-related cognitive dysfunction and prevents cholinergic neuron atrophy. J Neurosci 16:5437-5442.

Backman L, Small BJ, Wahlin A, Larsson M (2000) Cognitive functioning in very old age. In: The handbook of aging And Cognition (Craik FIM, Salthouse TA, eds), pp 499-558. Mahwah, NJ: Erlbaum.

Barnes CA (1994) Normal aging: regionally specific changes in hippocampal synaptic transmission. Trends Neurosci 17:13-18.

Barnes CA, McNaughton BL (1985) An age comparison of the rates of acquisition and forgetting of spatial information in relation to long-term enhancement of hippocampal synapses. Behav Neurosci 99:1040-1048.

Barnes CA, Rao G, Foster TC, McNaughton BL (1992) Region-specific age effects on AMPA sensitivity: electrophysiological evidence for loss of synaptic contacts in hippocampal field CA1. Hippocampus 2:457-468.

Barnes CA, Rao G, McNaughton BL (1996) Functional integrity of NMDA-dependent LTP induction mechanisms across the lifespan of F-344 rats. Learn Mem 3:124-137.

Barnes CA, Rao G, Shen J (1997) Age-related decrease in the N-methylD-aspartateR-mediated excitatory postsynaptic potential in hippocampal region CA1. Neurobiol Aging 18:445-452.

Billard JM, Jouvenceau A, Lamour Y, Dutar P (1997) NMDA receptor activation in the aged rat: electrophysiological investigations in the CA1 area of the hippocampal slice ex vivo. Neurobiol Aging 18:535-542.

Bliss TV, Lomo T (1973) Long-lasting potentiation of synaptic transmission in the dentate area of the anaesthetized rabbit following stimulation of the perforant path. J Physiol (Lond) 232:331-356.

Blitzer RD, Wong T, Nouranifar R, Iyengar R, Landau EM (1995) Postsynaptic cAMP pathway gates early LTP in hippocampal CA1 region. Neuron 15:1403-1414.

Blitzer RD, Connor JH, Brown GP, Wong T, Shenolikar S, Iyengar R,
Landau EM (1998) Gating of CaMKII by cAMP-regulated protein phosphatase activity during LTP. Science 280:1940-1942.

Bogdanovic N, Islam A, Nilsson L, Bergstrom L, Winblad B, Adem A (1993) Effects of nucleus basalis lesion on muscarinic receptor subtypes. Exp Brain Res 97:225-232.

Bowden SE, Fletcher S, Loane DJ, Marrion NV (2001) Somatic colocalization of rat SK1 and D class $\left(\mathrm{Ca}_{\mathrm{v}} 1.2\right)$ L-type calcium channels in rat CA1 hippocampal pyramidal neurons. J Neurosci 21:RC175(1-6).

Brown DA, Adams PR (1980) Muscarinic suppression of a novel voltage-sensitive $\mathrm{K}^{+}$current in a vertebrate neurone. Nature 283:673-676

Campbell LW, Hao SY, Thibault O, Blalock EM, Landfield PW (1996) Aging changes in voltage-gated calcium currents in hippocampal CA1 neurons. J Neurosci 16:6286-6295.

Clark AS, Magnusson KR, Cotman CW (1992) In vitro autoradiography of hippocampal excitatory amino acid binding in aged Fischer 344 rats: relationship to performance on the Morris water maze. Behav Neurosci 106:324-335.

Clayton DA, Browning MD (2001) Deficits in the expression of the NR2B subunit in the hippocampus of aged Fisher 344 rats. Neurobiol Aging 22:165-168.

Clayton DA, Mesches MH, Alvarez E, Bickford PC, Browning MD (2002) A hippocampal NR2B deficit can mimic age-related changes in long-term potentiation and spatial learning in the Fischer 344 rat. J Neurosci 22:3628-3637.

Davis HP, Tribuna J, Pulsinelli WA, Volpe BT (1986) Reference and working memory of rats following hippocampal damage induced by transient forebrain ischemia. Physiol Behav 37:387-392.

Davis S, Markowska AL, Wenk GL, Barnes CA (1993) Acetyl-L-carnitine: behavioral, electrophysiological, and neurochemical effects. Neurobiol Aging 14:107-115.

Deupree DL, Turner DA, Watters CL (1991) Spatial performance correlates with in vitro potentiation in young and aged Fischer 344 rats. Brain Res 554:1-9.

Deupree DL, Bradley J, Turner DA (1993) Age-related alterations in potentiation in the CA1 region in F344 rats. Neurobiol Aging $14: 249-258$.

Disterhoft JF, Thompson LT, Moyer Jr JR, Mogul DJ (1996) Calciumdependent afterhyperpolarization and learning in young and aging hippocampus. Life Sci 59:413-420.

Doods H, Entzeroth M, Ziegler H, Schiavi G, Engel W, Mihm G, Rudolf K, Eberlein W (1993a) Characterization of BIBN 99: a lipophilic and selective muscarinic M2 receptor antagonist. Eur J Pharmacol 242:23-30.

Doods HN, Quirion R, Mihm G, Engel W, Rudolf K, Entzeroth M, Schiavi GB, Ladinsky H, Bechtel WD, Ensinger HA, Medla KA, Eberlein W (1993b) Therapeutic potential of CNS-active M2 antagonists: novel structures and pharmacology. Life Sci 52:497-503.

Eckles-Smith K, Clayton D, Bickford P, Browning MD (2000) Caloric restriction prevents age-related deficits in LTP and in NMDA receptor expression. Mol Brain Res 78:154-162.

Foster TC (1999) Involvement of hippocampal synaptic plasticity in agerelated memory decline. Brain Res Rev 30:236-249.

Foster TC, Norris CM (1997) Age-associated changes in $\mathrm{Ca}^{2+}$. dependent processes: relation to hippocampal synaptic plasticity. Hippocampus 7:602-612.

Freund TF, Antal M (1988) GABA-containing neurons in the septum control inhibitory interneurons in the hippocampus. Nature 336:170-173.

Gage FH, Dunnett SB, Björklund A (1984) Spatial learning and motor deficits in aged rats. Neurobiol Aging 5:43-48.

Gulyas AI, Hajos N, Freund TF (1996) Interneurons containing calretinin are specialized to control other interneurons in the rat hippocampus. J Neurosci 16:3397-3411.

Hajos N, Papp EC, Acsady L, Levey AI, Freund TF (1998) Distinct interneuron types express $\mathrm{m} 2$ muscarinic receptor immunoreactivity on their dendrites or axon terminals in the hippocampus. Neuroscience 82:355-376

Hollup SA, Kjelstrup KG, Hoff J, Moser MB, Moser EI (2001) Impaired recognition of the goal location during spatial navigation in rats with hippocampal lesions. J Neurosci 21:4505-4513.

Kandel ER, Spencer WA (1961) Electrophysiology of hippocampal neurons. II. After-potentials and repetitive firing. $\mathrm{J}$ Neurophysiol 25:243-259

Kitaichi K, Hori T, Srivastava LK, Quirion R (1999) Antisense oligodeoxynucleotides against the muscarinic $\mathrm{m} 2$, but not $\mathrm{m} 4$, receptor supports its role as autoreceptors in the rat hippocampus. Mol Brain Res 67:98-106.

Krause M, Pedarzani P (2000) A protein phosphatase is involved in the cholinergic suppression of the $\mathrm{Ca}^{2+}$-activated $\mathrm{K}^{+}$current $\mathrm{SI}(\mathrm{AHP})$ in hippocampal pyramidal neurons. Neuropharmacology 39:1274-1283.

Krause M, Offermanns S, Stocker M, Pedarzani P (2002) Functional specificity of $G_{q}$ and $G_{11}$ in the cholinergic and glutamatergic modulation of potassium currents and excitability in hippocampal neurons. J Neurosci 22:666-673. 
Landfield PW, Pitler TA (1984) Prolonged $\mathrm{Ca}^{2+}$-dependent afterhyperpolarizations in hippocampal neurons of aged rats. Science 226:1089-1092.

Landfield PW, Pitler TA, Applegate MD (1986) The effects of high $\mathrm{Mg}^{2+}$-to-Ca ${ }^{2+}$ ratios on frequency potentiation in hippocampal slices of young and aged rats. J Neurophysiol 56:797-811.

Landfield PW, Campbell LW, Hao SY, Kerr DS (1989) Aging-related increases in voltage-sensitive, inactivating calcium currents in rat hippocampus. Implications for mechanisms of brain aging and Alzheimer's disease. Ann NY Acad Sci 568:95-105.

Levey AI, Kitt CA, Simonds WF, Price DL, Brann MR (1991) Identification and localization of muscarinic acetylcholine receptor proteins in brain with subtype-specific antibodies. J Neurosci 11:3218-3226.

Magnusson KR (2001) Influence of diet restriction on NMDA receptor subunits and learning during aging. Neurobiol Aging 22:613-627.

Marrion NV, Tavalin SJ (1998) Selective activation of $\mathrm{Ca}^{2+}$-activated $\mathrm{K}^{+}$channels by co-localized $\mathrm{Ca}^{2+}$ channels in hippocampal neurons. Nature 395:900-905.

Martin SJ, Grimwood PD, Morris RG (2000) Synaptic plasticity and memory: an evaluation of the hypothesis. Annu Rev Neurosci 23:649-711.

McClearn GE (1997) Biomarkers of age and aging. Exp Gerontol 32:87-94.

McEachern JC, Shaw CA (1999) The plasticity-pathology continuum: defining a role for the LTP phenomenon. J Neurosci Res 58:42-61.

Moore CI, Browning MD, Rose GM (1993) Hippocampal plasticity induced by primed burst, but not long-term potentiation, stimulation is impaired in area CA1 of aged Fischer 344 rats. Hippocampus 3:57-66.

Morris RGM, Garrud P, Rawlins JNP, O'Keefe J (1982) Place navigation is impaired in rats with hippocampal lesions. Nature 297:681-683.

Morris RGM, Schenk F, Tweedie F, Jarrard LE (1990) Ibotenate lesions of hippocampus and/or subiculum: dissociating components of allocentric spatial learning. Eur J Neurosci 2:1016-1028.

Moser M-B, Moser EI, Forrest E, Andersen P, Morris RGM (1995) Spatial learning with a minislab in the dorsal hippocampus. Proc Natl Acad Sci USA 92:9697-9701.

Moyer Jr JR, Thompson LT, Black JP, Disterhoft JF (1992) Nimodipine increases excitability of rabbit CA1 pyramidal neurons in an age- and concentration-dependent manner. J Neurophysiol 68:2100-2109.

Nguyen PV, Abel T, Kandel ER, Bourtchouladze R (2000) Straindependent differences in LTP and hippocampus-dependent memory in inbred mice. Learn Mem 7:170-179.

Norris CM, Korol DL, Foster TC (1996) Increased susceptibility to induction of long-term depression and long-term potentiation reversal during aging. J Neurosci 16:5382-5392.

Norris CM, Halpain S, Foster TC (1998a) Reversal of age-related alterations in synaptic plasticity by blockade of L-type $\mathrm{Ca}^{2+}$ channels. J Neurosci 18:3171-3179.

Norris CM, Halpain S, Foster TC (1998b) Alterations in the balance of protein kinase/phosphatase activities parallel reduced synaptic strength during aging. J Neurophysiol 80:1567-1570.

Otto T, Eichenbaum H, Wiener SI, Wible CG (1991) Learning-related patterns of CA1 spike trains parallel stimulation parameters optimal for inducing hippocampal long-term potentiation. Hippocampus 1:181-192.

Paulsen O, Sejnowski TJ (2000) Natural patterns of activity and longterm synaptic plasticity. Curr Opin Neurobiol 10:172-179.

Pike BP, Hamm RJ (1995) Post-injury administration of BIBN 99, a selective muscarinic M2 receptor antagonist, improves cognitive performance following traumatic brain injury in rats. Brain Res 686:37-43.

Pike FG, Meredith RM, Olding AW, Paulsen O (1999) Rapid report: postsynaptic bursting is essential for "Hebbian" induction of associative long-term potentiation at excitatory synapses in rat hippocampus. J Physiol (Lond) 518:571-576.

Pitler TA, Alger BE (1990) Activation of the pharmacologically defined
M3 muscarinic receptor depolarizes hippocampal pyramidal cells. Brain Res 534:257-262.

Quirion R, Wilson A, Rowe W, Aubert I, Richard J, Doods H, Parent A, White N, Meaney MJ (1995) Facilitation of acetylcholine release and cognitive performance by an $\mathrm{M}_{2}$-muscarinic receptor antagonist in aged memory-impaired rats. J Neurosci 15:1455-1462.

Rapp PR, Gallagher M (1996) Preserved neuron number in the hippocampus of aged rats with spatial learning deficits. Proc Natl Acad Sci USA 93:9926-9930.

Rapp PR, Stack EC, Gallagher M (1999) Morphometric studies of the aged hippocampus. I. Volumetric analysis in behaviorally characterized rats. J Comp Neurol 403:459-470.

Rouse ST, Edmunds SM, Yi H, Gilmor ML, Levey AI (2000) Localization of $\mathrm{M}(2)$ muscarinic acetylcholine receptor protein in cholinergic and non-cholinergic terminals in rat hippocampus. Neurosci Lett 284:182-186.

Rowe WB, Spreekmeester E, Meaney MJ, Quirion R, Rochford J (1998) Reactivity to novelty in cognitively-impaired and cognitivelyunimpaired aged rats and young rats. Neuroscience 83:669-680.

Sah P, Bekkers JM (1996) Apical dendritic location of slow afterhyperpolarization current in hippocampal pyramidal neurons: implications for the integration of long-term potentiation. J Neurosci 16:4537-4542.

Selcher JC, Weeber EJ, Sweatt JD (2000) Different molecular mechanisms for synaptic plasticity and learning in rats and mice. Soc Neurosci Abstr 26:467.16.

Shankar S, Teyler TJ, Robbins N (1998) Aging differentially alters forms of long-term potentiation in rat hippocampal area CA1. J Neurophysiol 79:334-341.

Shors TJ, Matzel LD (1997) Long-term potentiation: what's learning got to do with it? Behav Brain Sci 20:597-655.

Smith TD, Adams MM, Gallagher M, Morrison JH, Rapp PR (2000) Circuit-specific alterations in hippocampal synaptophysin immunoreactivity predict spatial learning impairment in aged rats. J Neurosci 20:6587-6593

Suzuki SS, Smith GK (1985) Burst characteristics of hippocampal complex spike cells in the awake rat. Exp Neurol 89:90-95.

Thomas MJ, Moody TD, Makhinson M, O'Dell TJ (1996) Activitydependent beta-adrenergic modulation of low frequency stimulation induced LTP in the hippocampal CA1 region. Neuron 17:475-482.

Thomas MJ, Watabe AM, Moody TD, Makhinson M, O'Dell TJ (1998) Postsynaptic complex spike bursting enables the induction of LTP by theta frequency synaptic stimulation. J Neurosci 18:7118-7126.

Toth K, Freund TF, Miles R (1997) Disinhibition of rat hippocampal pyramidal cells by GABAergic afferents from the septum. J Physiol (Lond) 500:463-474.

Tsien JZ, Chen DF, Gerber D, Tom C, Mercer EH, Anderson DJ, Mayford M, Kandel ER, Tonegawa S (1996) Subregion- and cell typerestricted gene knockout in mouse brain. Cell 87:1317-1326.

Tsubokawa H, Ross WN (1996) IPSPs modulate spike backpropagation and associated $\left[\mathrm{Ca}^{2+}\right]$ i changes in the dendrites of hippocampal CA1 pyramidal neurons. J Neurophysiol 76:2896-2906.

Tsubokawa H, Ross WN (1997) Muscarinic modulation of spike backpropagation in the apical dendrites of hippocampal CA1 pyramidal neurons. J Neurosci 17:5782-5791.

Vannucchi MG, Scali C, Kopf SR, Pepeu G, Casamenti F (1997) Selective muscarinic antagonists differentially affect in vivo acetylcholine release and memory performances of young and aged rats. Neuroscience 79:837-846.

Winder DG, Martin KC, Muzzio IA, Rohrer D, Chruscinski A, Kobilka B, Kandel ER (1999) ERK plays a regulatory role in induction of LTP by theta frequency stimulation and its modulation by beta-adrenergic receptors. Neuron 24:715-726.

Zhang Y, Carlen PL, Zhang L (1997) Kinetics of muscarinic reduction of IsAHP in hippocampal neurons: effects of acetylcholinesterase inhibitors. J Neurophysiol 78:2999-3007. 